\title{
Ankle impingement
}

\author{
Kyle P. Lavery ${ }^{*}(\mathbb{D}$, Kevin J. McHale, William H. Rossy and George Theodore
}

\begin{abstract}
Ankle impingement is a syndrome that encompasses a wide range of anterior and posterior joint pathology involving both osseous and soft tissue abnormalities. In this review, the etiology, pathoanatomy, diagnostic workup, and treatment options for both anterior and posterior ankle impingement syndromes are discussed.
\end{abstract}

Keywords: Ankle impingement, Ankle arthroscopy, Os trigonum

\section{Background}

Impingement refers to abnormal entrapment or contact of structures resulting in pain or restricted motion. Impingement syndromes are a commonly recognized source of musculoskeletal symptoms in many areas, notably subacromial impingement in the shoulder and femoroacetabular impingement in the hip. Similarly, impingement syndromes are an increasingly recognized source of pain and disability in the ankle.

Impingement syndromes in the ankle include a broad spectrum of pathology with varying etiologies, anatomic features, and presentations. Although no official classification exists, these syndromes are generally defined by the particular anatomic area involved. Specific anterior, anterolateral, anteromedial, posterior, posteromedial, posterolateral, and syndesmotic impingements have been described $[1,2]$. However, these pathologies are generally grouped into anterior and posterior impingement syndromes for simplicity.

Anterior ankle impingement syndrome results from compression of structures at the anterior margin of the tibiotalar joint during dorsiflexion. Anterior impingement has long been recognized as a cause of pain in athletes. In 1949, McMurray described "footballer's ankle", a commonly observed condition in professional soccer players involving anterior osteophytes of the dorsal talar neck and distal tibia. The term was later refined to "impingement exostoses" by O'Donoghue in 1957 to include other patient populations [3].

\footnotetext{
* Correspondence: Kplavery16@gmail.com

Division of Sports Medicine, Department of Orthopaedic Surgery,

Massachusetts General Hospital, Harvard Medical School, 175 Cambridge

Street, Suite 400, Boston, MA 02114, USA
}

Likened to a "nut in a nutcracker", posterior impingement syndrome is characterized by compression in the anatomic region between the posterior tibia and calcaneus during plantar flexion. While anatomists and surgeons have long recognized structures at risk for compression, such as the os trigonum, the operative treatment of posterior impingement was not reported until 1982 when Howse described treating a "posterior block of the ankle joint" in a population of elite dancers [4]. The presentation was later termed "talar compression syndrome" [5].

Ankle impingement is an increasingly recognized cause of symptoms in an athletic population as our understanding of the etiology, pathogenesis, and presentation continues to evolve. Recent advancements in diagnostic and treatment techniques aim to improve outcomes.

\section{Main text \\ Etiology and pathoanatomy Anterior impingement}

Anterior ankle impingement generally refers to entrapment of structures along the anterior margin of the tibiotalar joint in terminal dorsiflexion. Multiple osseous and soft tissue anatomic abnormalities have been recognized as causative factors.

Characteristic spurs or "exostoses" at the anterior distal tibia and dorsal talar neck have long been observed in athletes with anterior ankle pain and limited motion. Isolated talofibular lesions have also been described [6]. The morphology of anterior tibiotalar exostoses has been well-studied, and cadaveric dissections have found these lesions to be intra-articular, well within the distal tibial and dorsal talar capsular attachments $[7,8]$.

Although they are often referred to as "kissing osteophytes", these tibial and talar spurs surprisingly 
often do not actually overlap and abut. Evaluation of preoperative CT scans has shown that talar spurs generally lie medial to the midline of the talar dome and tibial spurs are generally located laterally [9]. A distinct trough in the articular talar dome often "accepts" the tibial osteophyte during dorsiflexion. Kim, et al. referred to this as a "tram-track lesion" [10], while Raikin, et al. termed it a "divot sign" [11]. Subsequent studies have confirmed a high rate of corresponding talar cartilage lesions (80.7 \%) and loose bodies in patients with distal tibial lesions [12].

Anterior intra-articular soft tissues may contribute to impingement in isolation or in conjunction with bony lesions. A triangular soft tissue mass composed primarily of adipose and synovial tissues exists in the anterior joint space. These tissues are compressed after $15^{\circ}$ of dorsiflexion in asymptomatic individuals [7]. Anterior osteophytes may limit the space available for this soft tissue and exacerbate its entrapment, resulting in chronic inflammation, synovitis, and capsuloligamentous hypertrophy. Post-traumatic fibrous bands [13], thickened anterior tibiofibular ligaments $[14,15]$, and synovial plica [16], have also been identified as causative factors.

While the impinging anatomic lesions have been well described, their exact etiology is less well understood. Early reports hypothesized spurs to be enthesophytes caused by traction to the anterior capsule during repetitive plantar flexion [3]. However, anatomic studies have demonstrated the chondral margins and lesions to be deep to the joint capsule rather than at its attachment, resulting in the traction, disproving the traction theory [7-9]. More recent observations of athletic populations commonly affected by anterior impingement have led to hypotheses that pathology occurs due to repetitive impaction injury to the anterior chondral margin from hyper-dorsiflexion or direct impact from an external object such as a soccer ball $[17,18]$.

Chronic lateral ankle instability has also been hypothesized to contribute to the development of both bony and soft tissue lesions associated with anterior impingement due to abnormal repetitive micromotion [14, 19]. Multiple studies have examined the prevalence of associated anterior impingement lesions at the time of arthroscopy in patients undergoing stabilization procedures for lateral ankle instability. Soft tissue lesions, such as synovitis in the anterior compartment or anterior lateral gutter, have been observed with high frequency (63-100\%), while anterior tibial osteophytes have often been found consistently (12-26.4 \%) [20-22]. In one study, patients undergoing a Brostrom procedure had 3.37 times the incidence of bone spurs than matched asymptomatic controls [23].

\section{Posterior impingement}

Posterior ankle impingement results from compression of structures posterior to the tibiotalar and talocalcaneal articulations during terminal plantar flexion. Similarly, this can be caused by multiple osseous and soft tissue etiologies in isolation or in combination.

Pathology associated with the lateral (trigonal) process of the posterior talus is the most common cause of posterior impingement (Fig. 1). Anatomic variants of this structure have been well described. A Stieda process refers to an elongated tubercle. An os trigonum may represent failure of fusion of a secondary ossification center to the talar body, although this structure has been heavily debated in the orthopedic and radiologic literature. Impingement related to the trigonal process can result from acute fracture, chronic injury due to repetitive microtrauma, or mechanical irritation of the surrounding soft tissues [24] (Fig. 2).

Less commonly, posterior symptoms may result from tibiotalar or subtalar degenerative joint disease due to osteophyte impingement or associated reactive hypertrophic capsule and synovium. Post-traumatic sequelae from fracture malunion of the posterior malleolus, talus, or calcaneus may also occur [25]. A case of talar osteonecrosis resulting in posterior impingement has also been reported [26].

Various soft tissue structures may cause posterior impingement symptoms as well. Posterior capsuloligamentous injury due to repetitive or acute hyperflexion can lead to inflammation, scarring and thickening of the capsule, posterior inferior tibiofibular ligament, and posterior fibers of the deltoid ligament [27-29] (Fig. 5). The flexor hallucis longus (FHL) tendon, running between the medial and lateral posterior processes of the talus, is commonly affected by tenosynovitis and tendinosis. The tendinopathy may result from overuse or secondary to irritation from surrounding abnormal bony anatomy. Anatomic soft tissue variants, such as the posterior intermalleolar ligament and several anomalous muscles, have been described as other sources of impingement [30-34].

\section{Presentation \\ Anterior impingement}

Anterior impingement syndrome typically presents as anterior ankle pain during terminal dorsiflexion. Exacerbating activities commonly include climbing stairs, running or walking up hills, ascending ladders, and deep squatting. The classic association with competitive soccer players has long been recognized, but the reason that this subset of athletes is commonly affected is unclear $[10,17,18]$. In the later stages, dorsiflexion may be limited secondary to mechanical block or pain, creating a cycle of progressive joint stiffness and loss of function. In isolated soft tissue lesions, the patient may report a subjective popping or snapping sensation.

\section{Posterior impingement}

Posterior impingement syndrome generally presents as a less specific pain deep to the Achilles tendon and may 


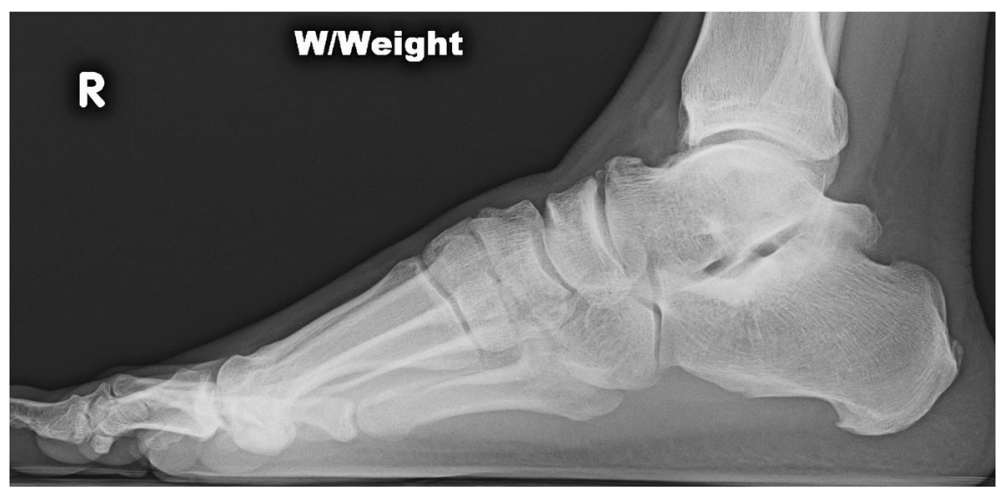

Fig. 1 A lateral radiograph demonstrates an elongated posterolateral (trigonal) process of the talus (Stieda process)

often be confused with Achilles or peroneal tendon pathology. Symptoms may be worsened by activities involving plantar flexion and repetitive push-off maneuvers, including downhill running and walking, descending stairs, and high-heeled shoe wear. Posterior impingement classically presents in dancers, specifically those participating in classic ballet, presumably due to repetitive weight bearing in the plantar-flexed "en-pointe" and "demi-pointe" positions [35-38]. In a recent systematic review, dancers represented $61 \%$ of patients undergoing surgery for posterior impingement [39]. It has also been reported to affect fast-bowlers in cricket [40].

\section{Physical examination}

A comprehensive physical examination of the foot and ankle should be performed when assessing for impingement syndromes. The ankle and foot are inspected for abnormal alignment, joint effusion, or soft tissue edema. The bone and soft tissue structures are systematically palpated to assess for localized tenderness. While anterior or anterolateral tenderness is characteristic in anterior impingement, posterior impingement signs can be more difficult to elicit and localize, as structures are deeper. Posteromedial ankle tenderness with resisted plantar flexion of the first metatarsophalangeal joint is more consistent with FHL pathology, while posterolateral tenderness with forced ankle plantar flexion is more likely to involve pathology associated with the trigonal process.

Passive and active ranges of motion of the joints bilaterally are measured, including dorsiflexion, plantar flexion, subtalar, and midfoot motions. Laterally, the peroneal tendon is assessed for tenderness, deformity, or subluxation. The sural nerve is evaluated for sensitivity. Posteriorly, the Achilles tendon is assessed for fusiform enlargement or retrocalcaneal bursitis. Medially, the tibial nerve is evaluated for tarsal tunnel syndrome, and the posterior tibial tendon's function is assessed. The anterior drawer and talar tilt tests of the tibiotalar joint are performed to exclude ankle instability. Finally, a straight leg raise test in the seated or supine position may be done to exclude an L5 or S1 radiculopathy.

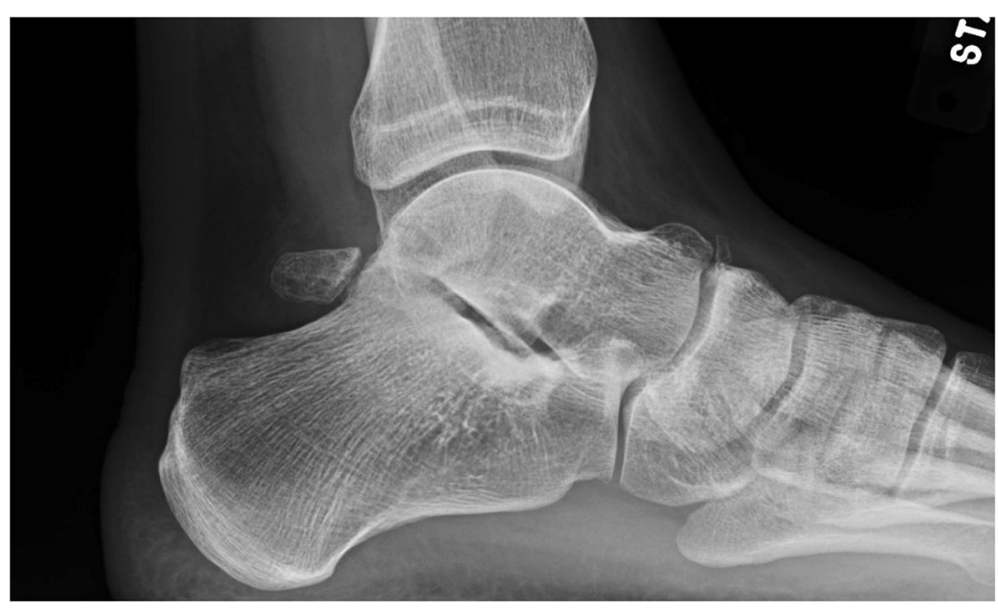

Fig. 2 A lateral radiograph demonstrates a large os trigonum 


\section{Imaging}

Imaging of an ankle suspected of impingement should begin with a plain $\mathrm{x}$-ray series, as the diagnosis is often confirmed with simple radiographs. Initial views should include weight-bearing AP, lateral, and mortise projections. Careful attention is given to the lateral view, assessing for exostoses on distal anterior tibia and dorsal talar neck and posterior bony abnormalities, including a Stieda process or os trigonum.

Alternative oblique views have been described for both anterior and posterior impingement lesions to better assess for bony abnormalities, as standard views can miss some lesions. To detect anteromedial lesions, the beam is aimed $45^{\circ}$ craniocaudad with the leg externally rotated $30^{\circ}$ [41]. The utility of the oblique anteromedial impingement view has been confirmed to have a higher sensitivity in detecting both tibial (85 vs. $40 \%$ ) and talar (73 vs. $32 \%$ ) osteophytes when added to a standard lateral radiograph [42]. Lesions associated with the trigonal process are best viewed on a $25^{\circ}$ external rotation-lateral view [43]. Dynamic hyper-plantar-flexed or dorsiflexed laterals can be considered to demonstrate abnormal bony contact.

Advanced imaging, such as MRI, may also be considered when the diagnosis remains inconclusive. Images should be evaluated for bone edema, effusion, synovitis, tenosynovitis, and concomitant chondral injury (Fig. 3). In anterior soft tissue impingement, the anterolateral gutter may contain hypertrophic synovium or fibrosis. Increased marrow signal intensity at the trigonal process or os trigonum is suggestive of an acute injury or chronic stress fracture [44]. The efficacy of MRI in evaluating soft tissue impingement lesions is variable,

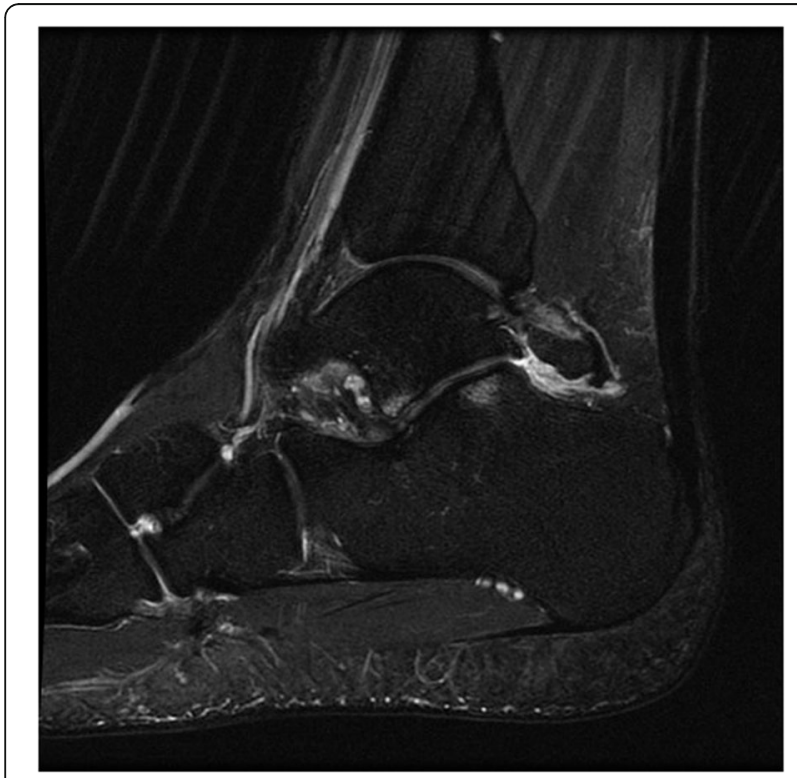

Fig. 3 A T2-weighted MRI with reactive edema surrounding an os trigonum with reported sensitivities 42-89\% and specificities 75-100\% [45-50]. Computed tomography has been used in defining the morphology of bony lesions for planning surgical resections [51]. Recently, ultrasound has also gained popularity as a reliable and inexpensive modality in evaluating impingement lesions and administering therapeutic injections $[52,53]$.

\section{Nonsurgical treatment}

Nonsurgical treatment remains the initial approach to the management of both anterior and posterior impingement syndromes, despite limited evidence of its efficacy. For acute symptoms, a period of rest and an avoidance of provocative activities are recommended. This approach can be supplemented with ice, NSAIDs, or cast immobilization in more severe cases. Rest can be supplemented with ice, NSAIDs, or immobilization in severe cases. In chronic cases, shoe modifications, including heel lift orthoses to prevent dorsiflexion, have been utilized. Physical therapy protocols focus on improving ankle stability and optimizing proprioception. Authors have reported successful symptom relief with ultrasound-guided corticosteroid injections, which may also have diagnostic uses [54, 55].

\section{Surgical treatment}

Surgical intervention is generally indicated for persistent symptoms which have not responded to non-operative treatment, affected normal activities of daily living or athletic performance, and correlated with physical exam and imaging findings. The surgical approach and technique vary by the anatomic region and pathology involved.

\section{Anterior impingement}

Surgical goals for the treatment of anterior impingement involve removing the offending pathologic lesion contributing to the symptoms. This may involve resection or debridement of bony lesions, soft tissue lesions, or both. Early studies described the use of open anterior or lateral arthrotomy [3]. A lateral arthrotomy is often still utilized if a lateral ligamentous procedure is being performed concurrently. However, open approaches have largely been replaced by arthroscopic techniques [56-80].

Hawkins is credited with reporting the first arthroscopic approach for the treatment of bony anterior ankle impingement in 1988, citing improved visualization with a less invasive approach [81]. Standard anterolateral and anteromedial portals are typically utilized and may be extended with conversion to open arthrotomy if necessary. An arthroscopic burr is used to reshape the anterior tibia and dorsal talus to their native contours. A combination of a shaver and electrothermal device is used to debride hypertrophic or inflamed synovium and fibrotic tissue (Fig. 4). Intraoperative fluoroscopy may be used to confirm adequate resection of spurs (Fig. 4). 

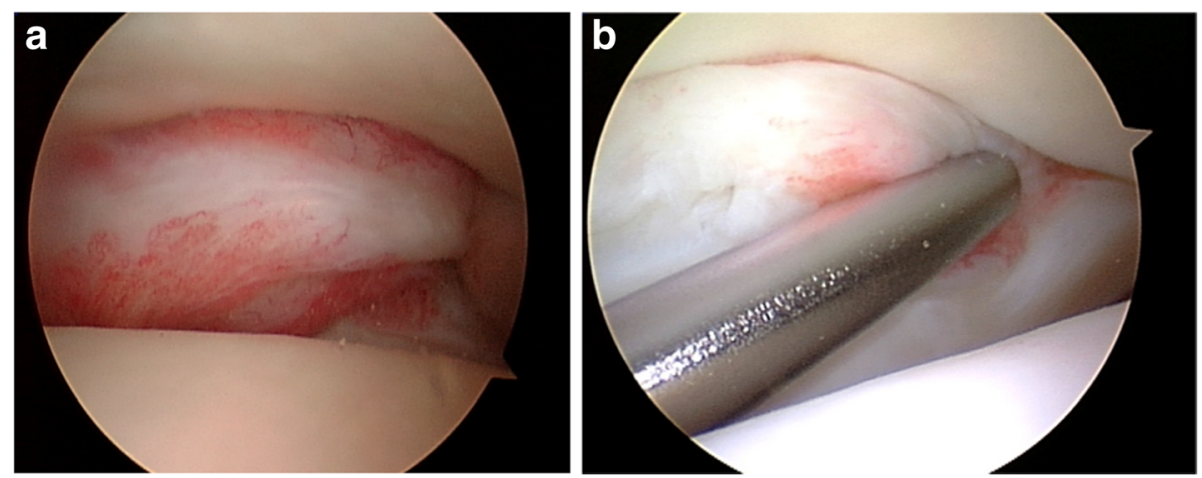

Fig. 4 a An arthroscopic image demonstrates anterolateral scar impingement with associated synovitis. b An arthroscopic shaver is used to resect the lesion

Zwiers et al. conducted a recent systematic review examining the results of the arthroscopic treatment of anterior impingement [75] (Fig. 5). The review included 19 studies and 905 patients, with an average age of 32.7 years. At a combined mean follow-up 35.3 months, $74-100 \%$ of patients were satisfied with the results of their procedure. AOFAS scores improved consistently, ranging from $34-75$ preoperatively and increasing to 83.5-92 postoperatively. There was a $5.1 \%$ overall complication rate, with $1.2 \%$ considered major complications. This is consistent with a $4 \%$ complication rate in a previous review by Simonson et al. [82].

\section{Posterior impingement}

Similarly, the surgical goal of treating posterior impingement involves resection of the causative anatomy. Most commonly, symptom relief is achieved by excision of a painful trigonal process or os trigonum, with debridement of surrounding inflammatory or hypertrophic soft tissues.

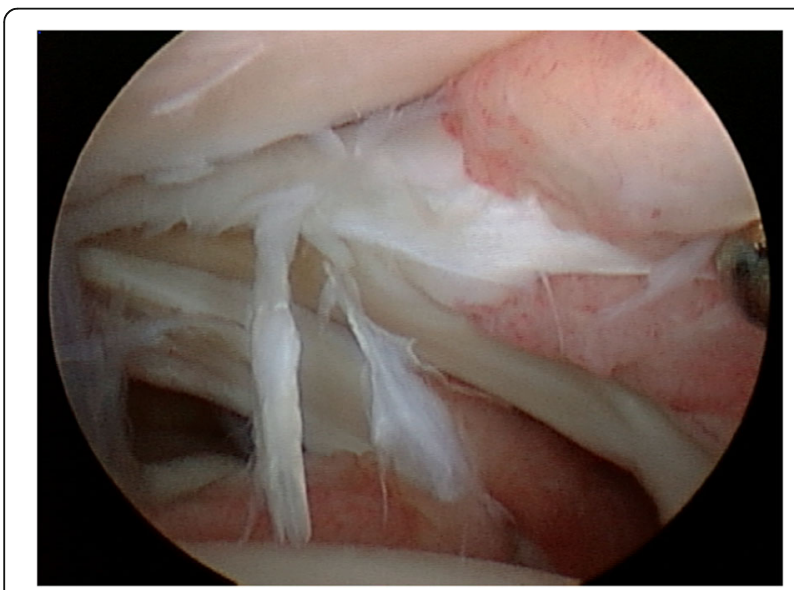

Fig. 5 An arthroscopic image demonstrates tearing of the posterior inferior tibiofibular ligament complex
Posterior pathology can be targeted through an open lateral, open medial, or endoscopic approach. A lateral approach allows for more direct access to the trigonal process with less risk to the medial neurovascular bundle. A medial approach allows for concomitant FHL pathology to be addressed more easily. Since 2000, posterior endoscopic approaches have gained popularity, with the potential for faster return to sport and lower complication rates [31, 83-97]. With the patient positioned prone, posteromedial and posterolateral hindfoot portals adjacent to the Achilles tendon typically provide excellent access to extra-articular posterior structures.

Ribbans et al. reviewed 47 papers consisting of 905 patients treated surgically with both open and endoscopic approaches for posterior impingement [39]. Eighty-one percent of symptoms were attributed to osseous pathology. In the included series, $67-100 \%$ of patients experienced good or excellent outcomes. Zwiers et al. conducted a similar systematic review including 16 studies [98]. Significantly lower complication rates (7.2 vs. $15.9 \%)$ and earlier return to full activity (11.3 vs. 16 weeks) were found with endoscopic surgery.

\section{Conclusions}

Ankle impingement can encompass a broad spectrum of anterior and posterior pathology involving both osseous and soft tissue abnormalities. While anterior impingement produces symptoms with terminal dorsiflexion, posterior impingement is exacerbated by activities involving hyper-plantar flexion. History, physical examination, imaging studies, and diagnostic injections all contribute to the accurate diagnosis of the condition. Many patients will respond favorably to non-operative treatment modalities, but both open and arthroscopic techniques have evolved to address chronic problems with successful and predictable outcomes. 


\section{Acknowledgements}

None.

\section{Funding}

Not applicable.

\section{Availability of data and materials}

Not applicable.

\section{Authors' contributions}

$\mathrm{KL}$ was the lead author of this manuscript. KM and WR were contributing authors. GT was the senior author. All authors read and approved the final manuscript

\section{Authors' information}

All the authors are members of the Division of Sports Medicine in the Department of Orthopaedic Surgery in Boston, MA

\section{Competing interests}

The authors declare that they have no competing interests.

\section{Consent for publication}

Not applicable.

\section{Ethics approval and consent to participate}

Not applicable.

Received: 26 May 2016 Accepted: 23 August 2016

Published online: 09 September 2016

\section{References}

1. Sanders TG, Rathur SK. Impingement syndromes of the ankle. Magn Reson Imaging Clin N Am. 2008;16(1):29-38, v.

2. Dimmick S, Linklater J. Ankle impingement syndromes. Radiol Clin N Am. 2013;51(3):479-510.

3. O'Donoghue DH. Impingement exostoses of the talus and tibia. J Bone Joint Surg Am. 1957;39-A(4):835-52. discussion, 852; passim.

4. Howse AJ. Posterior block of the ankle joint in dancers. Foot Ankle. 1982;3(2):81-4

5. Brodsky AE, Khalil MA. Talar compression syndrome. Am J Sports Med. 1986;14(6):472-6.

6. Ahn JY, Choi HJ, Lee WC. Talofibular bony impingement in the ankle. Foot Ankle Int. 2015;36(10):1150-5.

7. Tol JL, van Dijk CN. Etiology of the anterior ankle impingement syndrome: a descriptive anatomical study. Foot Ankle Int. 2004:25(6):382-6.

8. Hayeri MR, Trudell DJ, Resnick D. Anterior ankle impingement and talar bony outgrowths: osteophyte or enthesophyte? Paleopathologic and cadaveric study with imaging correlation. AJR Am J Roentgenol. 2009:193(4):W334-8.

9. Berberian WS, et al. Morphology of tibiotalar osteophytes in anterior ankle impingement. Foot Ankle Int. 2001;22(4):313-7.

10. Kim SH, Ha Kl, Ahn JH. Tram track lesion of the talar dome. Arthroscopy. 1999;15(2):203-6.

11. Raikin SM, Cooke PH. Divot sign: a new observation in anterior impingement of the ankle. Foot Ankle Int. 1999;20(8):532-3.

12. Moon JS, et al. Cartilage lesions in anterior bony impingement of the ankle. Arthroscopy. 2010;26(7):984-9.

13. Valkering KP, et al. "Web impingement" of the ankle: a case report. Knee Surg Sports Traumatol Arthrosc. 2013;21(6):1289-92.

14. Bassett 3rd FH, et al. Talar impingement by the anteroinferior tibiofibular ligament. A cause of chronic pain in the ankle after inversion sprain. J Bone Joint Surg Am. 1990;72(1):55-9.

15. Akseki D, et al. The distal fascicle of the anterior inferior tibio-fibular ligament as a cause of anterolateral ankle impingement: results of arthroscopic resection. Acta Orthop Scand. 1999:70(5):478-82.

16. Rosenbaum AJ, et al. Ankle impingement caused by an intra-articular plica: a report of 2 cases. Foot Ankle Spec. 2016:9(1):79-82.

17. Tol $J$, et al. The relationship of the kicking action in soccer and anterior ankle impingement syndrome. A biomechanical analysis. Am J Sports Med. 2002;30(1):45-50.
18. Massada JL. Ankle overuse injuries in soccer players. Morphological adaptation of the talus in the anterior impingement. J Sports Med Phys Fitness. 1991;31(3):447-51.

19. Cannon LB, Hackney RG. Anterior tibiotalar impingement associated with chronic ankle instability. J Foot Ankle Surg. 2000;39(6):383-6.

20. Lee J, Hamilton G, Ford L. Associated intra-articular ankle pathologies in patients with chronic lateral ankle instability: arthroscopic findings at the time of lateral ankle reconstruction. Foot Ankle Spec. 2011;4(5):284-9.

21. Hua $Y$, et al. Combination of modified Brostrom procedure with ankle arthroscopy for chronic ankle instability accompanied by intra-articular symptoms. Arthroscopy. 2010;26(4):524-8.

22. Odak S, et al. Arthroscopic evaluation of impingement and osteochondral lesions in chronic lateral ankle instability. Foot Ankle Int. 2015:36(9):1045-9.

23. Scranton Jr PE, McDermott JE, Rogers JV. The relationship between chronic ankle instability and variations in mortise anatomy and impingement spurs. Foot Ankle Int. 2000;21(8):657-64

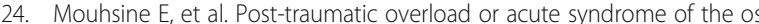
trigonum: a possible cause of posterior ankle impingement. Knee Surg Sports Traumatol Arthrosc. 2004:12(3):250-3.

25. Lui TH. Posterior ankle impingement syndrome caused by malunion of joint depressed type calcaneal fracture. Knee Surg Sports Traumatol Arthrosc. 2008;16(7):687-9.

26. Cortes ZE, Harris AM, Baumhauer JF. Posterior ankle pain diagnosed by positional MRI of the ankle: a unique case of posterior ankle impingement and osteonecrosis of the talus. Foot Ankle Int. 2006;27(4):293-5.

27. Koulouris $\mathrm{G}$, et al. Posterior tibiotalar ligament injury resulting in posteromedial impingement. Foot Ankle Int. 2003;24(8):575-83.

28. Paterson RS, Brown JN. The posteromedial impingement lesion of the ankle. A series of six cases. Am J Sports Med. 2001;29(5):550-7.

29. Peace $K A$, et al. MRI features of posterior ankle impingement syndrome in ballet dancers: a review of 25 cases. Clin Radiol. 2004;59(11):1025-33.

30. Fiorella D, Helms CA, Nunley 2nd JA. The MR imaging features of the posterior intermalleolar ligament in patients with posterior impingement syndrome of the ankle. Skelet Radiol. 1999:28(10):573-6.

31. Lohrer H, Arentz S. Posterior approach for arthroscopic treatment of posterolateral impingement syndrome of the ankle in a top-level field hockey player. Arthroscopy. 2004;20(4):e15-21.

32. Rosenberg ZS, et al. Posterior intermalleolar ligament of the ankle: normal anatomy and MR imaging features. AJR Am J Roentgenol. 1995;165(2):387-90.

33. Best $A$, et al. Posterior impingement of the ankle caused by anomalous muscles. A report of four cases. J Bone Joint Surg Am. 2005;87(9):2075-9.

34. Seipel $\mathrm{R}$, et al. The peroneocalcaneus internus muscle: an unusual cause of posterior ankle impingement. Foot Ankle Int. 2005;26(10):890-3.

35. Moser BR. Posterior ankle impingement in the dancer. Curr Sports Med Rep. 2011;10(6):371-7.

36. Hamilton WG, Geppert MJ, Thompson FM. Pain in the posterior aspect of the ankle in dancers. Differential diagnosis and operative treatment. J Bone Joint Surg Am. 1996;78(10):1491-500

37. Russell JA, et al. Pathoanatomy of posterior ankle impingement in ballet dancers. Clin Anat. 2010;23(6):613-21

38. Russell JA, et al. Pathoanatomy of anterior ankle impingement in dancers. J Dance Med Sci. 2012;16(3):101-8.

39. Ribbans WJ, et al. The management of posterior ankle impingement syndrome in sport: a review. Foot Ankle Surg. 2015;21(1):1-10.

40. Mansingh A. Posterior ankle impingement in fast bowlers in cricket. West Indian Med J. 2011;60(1):77-81.

41. van Dijk CN, et al. Oblique radiograph for the detection of bone spurs in anterior ankle impingement. Skelet Radiol. 2002;31(4):214-21.

42. Tol $\mathrm{JL}$, et al. The anterior ankle impingement syndrome: diagnostic value of oblique radiographs. Foot Ankle Int. 2004;25(2):63-8.

43. Wiegerinck Jl, et al. The posterior impingement view: an alternative conventional projection to detect bony posterior ankle impingement. Arthroscopy. 2014;30(10):1311-6.

44. Bureau NJ, et al. Posterior ankle impingement syndrome: MR imaging findings in seven patients. Radiology. 2000;215(2):497-503.

45. Huh YM, et al. Synovitis and soft tissue impingement of the ankle: assessment with enhanced three-dimensional FSPGR MR imaging. J Magn Reson Imaging. 2004;19(1):108-16.

46. Duncan $\mathrm{D}$, et al. The usefulness of magnetic resonance imaging in the diagnosis of anterolateral impingement of the ankle. J Foot Ankle Surg. 2006:45(5):304-7. 
47. Farooki S, Yao L, Seeger LL. Anterolateral impingement of the ankle: effectiveness of MR imaging. Radiology. 1998;207(2):357-60.

48. Schaffler GJ, et al. Impingement syndrome of the ankle following supination external rotation trauma: MR imaging findings with arthroscopic correlation. Eur Radiol. 2003;13(6):1357-62.

49. Lee JW, et al. Soft tissue impingement syndrome of the ankle: diagnostic efficacy of MRI and clinical results after arthroscopic treatment. Foot Ankle Int. 2004;25(12):896-902.

50. Ferkel RD, et al. MRI evaluation of anterolateral soft tissue impingement of the ankle. Foot Ankle Int. 2010;31(8):655-61.

51. Takao M, et al. Arthroscopic treatment for anterior impingement exostosis of the ankle: application of three-dimensional computed tomography. Foot Ankle Int. 2004;25(2):59-62.

52. McCarthy $\mathrm{CL}$, Wilson DJ, Coltman TP. Anterolateral ankle impingement: findings and diagnostic accuracy with ultrasound imaging. Skelet Radiol. 2008;37(3):209-16

53. Pesquer $\mathrm{L}$, et al. US in ankle impingement syndrome. J Ultrasound. 2014;17(2):89-97.

54. Jose J, et al. Sonographically guided therapeutic injections in the meniscoid lesion in patients with anteromedial ankle impingement syndrome. Foot Ankle Spec. 2014;7(5):409-13.

55. Robinson P, Bollen SR. Posterior ankle impingement in professional soccer players: effectiveness of sonographically guided therapy. AJR Am J Roentgenol. 2006;187(1):W53-8.

56. Bauer T, Breda R, Hardy P. Anterior ankle bony impingement with joint motion loss: the arthroscopic resection option. Orthop Traumatol Surg Res. 2010;96(4):462-8.

57. Ferkel RD, et al. Arthroscopic treatment of anterolateral impingement of the ankle. Am J Sports Med. 1991;19(5):440-6.

58. Baums $\mathrm{MH}$, et al. Clinical outcome of the arthroscopic management of sports-related "anterior ankle pain": a prospective study. Knee Surg Sports Traumatol Arthrosc. 2006;14(5):482-6.

59. Meislin RJ, et al. Arthroscopic treatment of synovial impingement of the ankle. Am J Sports Med. 1993:21(2):186-9.

60. Ogilvie-Harris DJ, Mahomed N, Demaziere A. Anterior impingement of the ankle treated by arthroscopic removal of bony spurs. J Bone Joint Surg (Br). 1993;75(3):437-40

61. Liu SH, et al. Arthroscopic treatment of anterolateral ankle impingement. Arthroscopy. 1994;10(2):215-8.

62. Reynaert P, Gelen G, Geens G. Arthroscopic treatment of anterior impingement of the ankle. Acta Orthop Belg. 1994;60(4):384-8.

63. Branca $\mathrm{A}$, et al. Arthroscopic treatment of anterior ankle impingement. Foot Ankle Int. 1997;18(7):418-23.

64. DeBerardino TM, Arciero RA, Taylor DC. Arthroscopic treatment of soft-tissue impingement of the ankle in athletes. Arthroscopy. 1997;13(4):492-8.

65. van Dijk CN, Tol JL, Verheyen CC. A prospective study of prognostic factors concerning the outcome of arthroscopic surgery for anterior ankle impingement. Am J Sports Med. 1997;25(6):737-45.

66. Kim SH, Ha Kl. Arthroscopic treatment for impingement of the anterolateral soft tissues of the ankle. J Bone Joint Surg (Br). 2000;82(7):1019-21.

67. Tol JL, Verheyen CP, van Dijk CN. Arthroscopic treatment of anterior impingement in the ankle. J Bone Joint Surg (Br). 2001;83(1):9-13.

68. Rasmussen $\mathrm{S}$, Hjorth Jensen $\mathrm{C}$. Arthroscopic treatment of impingement of the ankle reduces pain and enhances function. Scand J Med Sci Sports. 2002;12(2):69-72.

69. Nihal A, Rose DJ, Trepman E. Arthroscopic treatment of anterior ankle impingement syndrome in dancers. Foot Ankle Int. 2005;26(11):908-12.

70. Urguden $\mathrm{M}$, et al. Arthroscopic treatment of anterolateral soft tissue impingement of the ankle: evaluation of factors affecting outcome. Arthroscopy. 2005;21(3):317-22

71. Moustafa El-Sayed AM. Arthroscopic treatment of anterolateral impingement of the ankle. J Foot Ankle Surg. 2010;49(3):219-23.

72. Murawski CD, Kennedy JG. Anteromedial impingement in the ankle joint: outcomes following arthroscopy. Am J Sports Med. 2010;38(10):2017-24.

73. Arnold $\mathrm{H}$. Posttraumatic impingement syndrome of the ankle-indication and results of arthroscopic therapy. Foot Ankle Surg. 2011;17(2):85-8.

74. Brennan SA, et al. Arthroscopic debridement for soft tissue ankle impingement. Ir J Med Sci. 2012;181(2):253-6.

75. Zwiers R, et al. Arthroscopic treatment for anterior ankle impingement: a systematic review of the current literature. Arthroscopy. 2015;31(8):1585-96.
76. Buda R, et al. Arthroscopic treatment and prognostic classification of anterior soft tissue impingement of the ankle. Foot Ankle Int. 2016:37(1):33-9.

77. Gulish HA, Sullivan RJ, Aronow M. Arthroscopic treatment of soft-tissue impingement lesions of the ankle in adolescents. Foot Ankle Int. 2005;26(3):204-7.

78. Mardani-Kivi M, et al. Arthroscopic treatment of patients with anterolateral impingement of the ankle with and without chondral lesions. J Foot Ankle Surg. 2013;52(2):188-91.

79. Parma A, et al. Arthroscopic treatment of ankle anterior bony impingement: the long-term clinical outcome. Foot Ankle Int. 2014;35(2):148-55.

80. Rouvillain JL, et al. Distraction-free ankle arthroscopy for anterolateral impingement. Eur J Orthop Surg Traumatol. 2014;24(6):1019-23.

81. Hawkins RB. Arthroscopic treatment of sports-related anterior osteophytes in the ankle. Foot Ankle. 1988;9(2):87-90.

82. Simonson DC, Roukis TS. Safety of ankle arthroscopy for the treatment of anterolateral soft-tissue impingement. Arthroscopy. 2014;30(2):256-9.

83. van Dijk CN, de Leeuw PA, Scholten PE. Hindfoot endoscopy for posterior ankle impingement. Surgical technique. J Bone Joint Surg Am. 2009;91 Suppl 2:287-98.

84. van Dijk CN, Scholten PE, Krips R. A 2-portal endoscopic approach for diagnosis and treatment of posterior ankle pathology. Arthroscopy. 2000:16(8):871-6.

85. Tey $\mathrm{M}$, et al. Benefits of arthroscopic tuberculoplasty in posterior ankle impingement syndrome. Knee Surg Sports Traumatol Arthrosc. 2007;15(10):1235-9.

86. Scholten PE, Sierevelt IN, van Dijk CN. Hindfoot endoscopy for posterior ankle impingement. J Bone Joint Surg Am. 2008;90(12):2665-72.

87. Willits $\mathrm{K}$, et al. Outcome of posterior ankle arthroscopy for hindfoot impingement. Arthroscopy. 2008;24(2):196-202.

88. Calder JD, Sexton SA, Pearce CJ. Return to training and playing after posterior ankle arthroscopy for posterior impingement in elite professional soccer. Am J Sports Med. 2010;38(1):120-4.

89. Noguchi $\mathrm{H}$, et al. Arthroscopic excision of posterior ankle bony impingement for early return to the field: short-term results. Foot Ankle Int. 2010;31(5):398-403.

90. Sundararajan PP. Combined arthroscopic and fluoroscopic guidance in the atraumatic treatment of posterior ankle impingement syndrome. J Foot Ankle Surg. 2012:51(5):687-9.

91. Smyth NA, et al. Hindfoot arthroscopic surgery for posterior ankle impingement: a systematic surgical approach and case series. Am J Sports Med. 2013;41(8):1869-76.

92. Vila J, et al. Hindfoot endoscopy for the treatment of posterior ankle impingement syndrome: a safe and reproducible technique. Foot Ankle Surg. 2014;20(3):174-9.

93. Lui TH. Arthroscopic management of posteromedial ankle impingement. Arthrosc Tech. 2015;4(5):e425-7.

94. Miyamoto W, Takao M, Matsushita T. Hindfoot endoscopy for posterior ankle impingement syndrome and flexor hallucis longus tendon disorders. Foot Ankle Clin. 2015;20(1):139-47.

95. Carreira DS, et al. Outcome of arthroscopic treatment of posterior impingement of the ankle. Foot Ankle Int. 2016:37(4):394-400.

96. Dinato $\mathrm{MC}$, et al. Endoscopic treatment of the posterior ankle impingement syndrome on amateur and professional athletes. Knee Surg Sports Traumatol Arthrosc. 2016;24(4):1396-401.

97. Galla M, Lobenhoffer P. Technique and results of arthroscopic treatment of posterior ankle impingement. Foot Ankle Surg. 2011;17(2):79-84.

98. Zwiers $\mathrm{R}$, et al. Surgical treatment for posterior ankle impingement. Arthroscopy. 2013:29(7):1263-70. 